\title{
Research on Image Denoising with Wavelet Transform and Finite Element Method
}

\author{
Zeng Tai-sheng \\ Faculty of Mathematics and Computer Science \\ Quanzhou Normal University, Fujian, Quanzhou, China \\ 407305203@qq.com
}

\begin{abstract}
Image denoising is the modern problems often encountered in the image processing technology, the denoising algorithm puts forward in this paper is mainly using the characteristics that wavelet transform is suitable for processing point strange and finite element transformation line is suitable for processing some strange, and adopt the method of image partition to separate the image into homogeneous blocks and non homogenous blocks, use wavelet transform to process the homogenous blocks, and finite element method processes non homogenous block, the experimental results show that this method has better denoising effect, should have good practical value.
\end{abstract}

Keywords: Image denoising; wavelet transform; finite element; image partition

\section{Introduction}

In the real world, the image is an important source of access to information. According to statistics, about $75 \%$ of the information is obtained through the visual system. This is because an image contains and visual information is unmatched by other ways, such as voice, text. Before image analysis and use, generally go through strict pretreatment process, such as enhanced, denoising, quantitative, compression coding, etc. This paper mainly studies image denoising. Through image sensor will be useful in the real world image signal sampling, quantization, coding, transmission, in the process of recovery, there are many factors affecting the quality of the image, the noise is an important cause of image quality lower, the image in the process of acquisition, conversion and transmission, often by imaging equipment, transmission equipment as well as external environment noise influence, therefore, the actual image obtained by some commonly contain noise interference. The main source of noise with atmospheric disturbance, electromagnetic interference, camera device and photoelectric conversion device noise, the noise seriously reduce the quality of the image signal. And in the field of scientific research, production, national defense, and many other, more and more high to the requirement of image quality, many image processing also need higher quality images, in this case, the people have to from various angles to explore in order to improve the quality of the image.

Noise processing is a very important role in image processing. Image is often random signal intensity (can also be called "noise") by pollution. In order to make the image more vivid, the need for image denoising. How to reasonably to eliminate a lot of noise, to keep the image of the original of all kinds of information, and to strengthen, and suitable for the human eye observation, has always been a image processing one of the main topics of the research neighborhood. With the development of computer technology, to improve the performance of the CPU, the popularity of large screen display, people is higher and higher demand for all kinds of video image, so for the image denoising technology demands more and more is also high. How can get better results in a shorter period of time, the resolution of the larger image has always been of concern to people. 
Image denoising in other ways also have many applications, such as image recognition, in the presence of various interference information, accurate to find key information contains noise in the image, and lay a foundation for the subsequent processing of the image, such as applications in remote sensing images, aerial image, and image monitoring.

In the traditional image denoising neighborhood, the thinking of image denoising is divided into two main direction, using the image spatial domain information in image denoising, or derived from the Angle of the signal, the image as a two-dimensional signal, the signal processing in the frequency domain for image denoising. In spatial domain image denoising, mainly around the pixel to be processed image information is used to estimate calculation, including bilateral denoising method is relatively classic, in many cases have good performance, but any way could not adapt to all of the situation, it also has many problems. Since it is a kind of linear denoising method, there is a loss of details and inevitable defects such as fuzzy boundaries too.

After bilateral denoising algorithm, Buades and proposed a new better denoising algorithm, called the Non Local Means denoising algorithm, referred to as "while NLM algorithm, and can be referred to as the Local finite element denoising algorithm, this algorithm will bilateral denoising is calculated in the object from the level of pixel to pixel level, which USES pixels around the whole piece of image information to represent the point of information, so that we can effectively reduce the large amount of noise under the condition of the center pixel is not credible. And can be seen from the name of the algorithm, its calculation is local, theoretically, should be in full search more similar pieces of information, but in the actual calculation process, is similar to the one based on image block distribution is concentrated this assumption, of the similar area in the process of the search is still limited in a reasonable range.

At present, the existing image denoising methods are mostly based on the characteristics of the original image, the noise probability function etc to analyze noise and spectrum characteristics, etc. In both the noise spectrum and frequency domain signal without overlapping, low-frequency filtering method is very effective. When white noise signal containing noise, the frequency spectrum of the signal and noise can have overlap, the low frequency filter method denoising effect is not ideal, namely it in removing noise at the same time, also filter out the part of the edge information of original image, the image edge is relatively fuzzy, but if can effectively keep the edge details of the high frequency filter leads to expand outside noise consequences, denoising effect is not good. Related workers have proposed many multiple method of noise filtering the image, this paper adopts the method is to filter of wavelet image denoising and mean filtering and improve it. Finite element application of filter to remove impulse noise effect is more ideal, and the wavelet filter filter gaussian noise, the result is satisfactory, but when multiple noise respectively used to filter out alone, the resulting effect cannot but it is very good. After a great deal of analysis and simulation experiment, the average filtering and wavelet filtering with the combination of multiple filtering algorithm is effective and feasible.

\section{Related Works}

For a lot of noise more outstanding points, they are some of the gray level is only for different noise. Their distribution, and spread them with diffusion equation can be (is actually changing the point for the noise pixel values, make its gray and gray contrast in the perimeter points, make the human eye is difficult to distinguish, or the machine can also be difficult to distinguish (within the permissible standard in artificially) or make noise greatly abate). Of course this diffusion is the use of the gray levels of the perimeter points to change the grey value of noise points, to take the perimeter points method also has a lot of (neighbourhood domain method and determining the eight neighborhood). Of 
course, if the filter radius (window) is large, for larger noise can be more effective to remove, but has the potential to more severe fuzzy boundaries. For different noise, of course, need to use a different model, because up to now, no general denoising model. For noise image, first of all, probably to analyze its noise type, and then adopt corresponding model (for those who can know in advance which model noise effectively, but may not be from the nature of the mathematical properties of the model itself and noise to analyze which kind of model to which effective noise), and of course for mixed noise, can be used to combine multiple models, so far, what kinds of noise is not what the nature of models to standard or conclusion.

According to the image and noise superimposition relationship, noise can be divided into multiplicative noise and additive noise and additive noise has nothing to do with the input image signal, multiplicative noise and image signal, in this article generally considered the additive noise. Common additive white gaussian noise and salt and pepper noise (or called the impulse noise), among them, the amplitude and power spectral density is uniformly distributed random distribution on the noise is gaussian white noise; Have essentially the same amplitude, but distribution random noise is salt and pepper noise.

\subsection{Finite Element Algorithm based on Impulse Noise Detection}

Finite element is a field of pixel operation window pixels in order of size in the middle of the value as the output value to the center pixel. This article uses the method is to detect all possible first impulse noise points, then use filter window is not impulse noise pollution in the pixel gray value, as the center pixel gray value estimate. Due to the impulse noise identification process in advance, even if the pulse noise pollution is heavier, the finite element method based on impulse noise detection, also can get better filtering results.

Given the definition of correlation windows [1.2]: $\mathrm{C}$ is window for noise detection; $\mathrm{B}$ is the filter window; $\left[\mathrm{X}_{m n}\right]$ said the image of the corresponding matrix; $\mathrm{C}\left[\mathrm{X}_{m n}\right]$ is point $x m n$ operation center of the window. By the literature [3], the measuring point window contains a pixel point xmn centered $(2 n+1) *(2 n+1)$ pixels, then the window all the points in the mean and standard deviation are:

$$
\begin{gathered}
x_{m n}^{\prime}=\frac{1}{N^{2}} \sum_{i=1}^{N} \sum_{j=1}^{N} x_{i j} \\
a_{m n}=\sqrt{\frac{\sum_{i=1}^{N} \sum_{j=1}^{N}\left(x_{i j}-x_{i j}^{\prime}\right)^{2}}{(N-1)^{2}}}
\end{gathered}
$$

Image pixel gray value is always a part with the surrounding pixels continuous gradient, when the noise appeared on the original pixels, which is due to its pixel, on a convex concave under as impulse noise point on the minimum or maximum of gray value of image, and then its neighborhood gray scale of pixels within the gap is bigger. Thus, according to the continuity of image grayscale and impulse noise jump degeneration, the original pixels and impulse noise points available to distinguish and identify. If again according to probability theory, $\rho$ probability density for the impulse noise, the noise of grey value of positive and negative probability are $\rho / 2$. Therefore, in order to detect the noise more efficiently, can inspect point $x_{i j}$ specific size of the grey value. If you point $x_{i j}$ grey value of more than one parameter $\mathrm{t}$, could be the noise points. That is, $x_{i j} \in[0, \mathrm{t}]$ or[255-t,255]. According to the experience of the simulation, it shows that the noise pixel grayscale range between $[0,10]$ and $[245,255]$, so the parameter $t$ value in the range of $(5,10]$. 
For impulse noise detection and identification, combined with the literature [10], using a simple and rapid and practical effective impulse noise detection method, which satisfies the conditions (3), the point is xmn impulse noise point, the opposite is not:

$$
\left\{\begin{array}{l}
\left|x_{m n}-x_{m n}^{\prime}\right|>k \times a_{m n} \\
0 \leq x_{m n} \leq t \\
255 \geq x_{m n} \geq 255-t
\end{array}\right.
$$

among them, $x_{m n}^{\prime}$ and $a_{m n}$ respectively are the pixel values of the mean and standard deviation within $\mathrm{C}, \mathrm{k}$ is constant threshold, and $\mathrm{k}>0, \mathrm{t}$ as gray parameters, and $t \in[5,10]^{[4-5]}$

For $\left[\mathbf{X}_{m n}\right]$ impulse noise point distribution, define a binary identity matrix whose dimension is the same as the original image of define a and the dimensions of the original image size of the same binary pulse noise identification matrix $\left(\mathrm{B}_{\mathrm{mn}}\right)^{[6]}$. If the noise in the identity matrix $\mathrm{Bmn}=0$, it corresponds to the pixel is impulse noise point in the original image; If the noise identification matrix $\mathrm{B}_{\mathrm{mn}}=1$, prove this point is not polluted in the original image pixels. Initialize all the elements in the matrix $\left(\mathrm{B}_{\mathrm{mn}}\right)$ and is set to 1 , impulse noise detection, on the basis of the result to its elements assignment, the income of a binary image $\left(\mathrm{B}_{\mathrm{mn}}\right)$, reflects the distribution of impulse noise.

Specific impulse noise filtering method is: first, choose the appropriate D filtering window, select the dimension of $3 \times 3$. In the impulse noise identification matrix $\left(B_{m n}\right)$, to identify 1 point does not make processing, identification of 0 point processing by the following formula ${ }^{[7]}$ :

$$
\mathrm{y}_{\mathrm{mn}}=\left\{\begin{array}{l}
\left.M\left(\mathrm{q}_{\mathrm{x}}\right]\right), \quad \mathrm{B}_{\mathrm{mn}}=0 \text { and } \mathrm{s} \text { is odd number } \\
\left.\left.\left\{M_{1}\left(\mathrm{Q}_{m n}\right]\right)+M_{2}\left(\mathrm{q} \mathrm{x}_{m n}\right]\right)\right\} / 2, \quad \mathrm{~B}_{\mathrm{mn}}=0 \text { and } \mathrm{s} \text { is even number } \\
\mathrm{x}_{m n}, \quad \mathrm{~B}_{\mathrm{mn}}=1 \text { or } \mathrm{s}=0
\end{array}\right.
$$

among them, $s$ is number of signal points in the filter window is $\mathrm{D}$, you have an odd number of signal points in $\left.M\left(\mathrm{Q}_{m n}\right]\right)$ to $\mathrm{D}$ pixel values in order of size when the median, $\left.M_{1}\left(\mathrm{q}_{\mathrm{x}_{m n}}\right]\right)$ and $\left.M_{2}\left(\mathrm{q}_{\mathrm{x}_{m n}}\right]\right)$ to $\mathrm{D}$ have an even number of signal points in pixel value according to the size of the order in the middle of the two values, $\mathrm{y}_{\mathrm{mn}}$ is $\mathrm{x}_{m n}$ pixel values of the processed.

For the processed the identity of the matrix $B_{\mathrm{mn}}^{\prime}$ calculated by the following formula ${ }^{[8]}$ :

$$
B_{\mathrm{mn}}^{\prime}= \begin{cases}1, & \mathrm{y}_{m n} \neq x_{m n} \\ B_{m n}, & \mathrm{y}_{m n}=x_{m n}\end{cases}
$$

After finite element can be obtained by the algorithm of matrix $\left[\mathrm{y}_{m n}\right]$, also need to determine whether the binary matrix $B_{\mathrm{mn}}^{\prime}$, after surviving the noise of the logo is 0 points. If there is the matrix $\left[\mathrm{y}_{m n}\right]$, then repeat the above steps, until the binary pulse noise identifier with no longer exist in matrix $B_{\mathrm{mn}}^{\prime}$ zero point. At this point, the image $\left[\mathrm{y}_{m n}\right]$ is whole the output of finite element algorithm. 


\subsection{Based on Noise Estimation of Two-dimensional Fractional Wavelet Transform Filter Algorithm}

Fractional wavelet transform multi-resolution analysis of wavelet transform theory to promote the fractional Fourier domain, its associated with the evolution of the wavelet transform and the theory of fractional order, comprehensive characteristics of the two came into being at the same time, is a new kind of time and frequency domain analysis method. Compared with the wavelet transform, the biggest advantages of fractional wavelet transform added a variable $p$ order of time, can be more flexible to adjust the wavelet coefficients. Good application prospects of fractional wavelet transform and the signal processing method based on fractional wavelet transform the potential applications of self-evident.

Secondary fractional order wavelet transform are defined as shown below:

$$
W\left(\mathrm{a}_{m n}, \mathrm{~b}^{\prime}\right)=\iiint \int B_{\rho 1, \rho 2}\left(\mathrm{x}, \mathrm{y}, \mathrm{x}^{\prime}, \mathrm{y}^{\prime}\right) \mathrm{f}(\mathrm{x}, \mathrm{y}) \times \mathrm{h}_{\mathrm{a}_{m n}, \mathrm{~b}^{\prime}}\left(\mathrm{x}^{\prime}, \mathrm{y}^{\prime}\right) \mathrm{dxdydx} \mathrm{x}^{\prime} \mathrm{y}^{\prime}
$$

Fractional domain is expressed as:

$$
\begin{gathered}
W\left(\mathrm{a}_{m n}, \mathrm{~b}^{\prime}\right)=\left(\mathrm{a}_{m} \mathrm{a}_{n}\right)^{0.5} \iint H\left(\mathrm{a}_{m} \mu, \mathrm{a}_{n} v\right) \times \exp \left(\mathrm{j} 2 \pi \mu b_{\mathrm{x}^{\prime}}, \mathrm{j} 2 \pi v \mathrm{~b}_{\mathrm{y}^{\prime}}\right) \\
\times \mathrm{F}\left\{F^{\phi 1, \phi 2}[\mathrm{~F}(\mathrm{x}, \mathrm{y})]\left(\mathrm{x}^{\prime}, \mathrm{y}^{\prime}\right)\right\}(\mu, v) \mathrm{d} \mu \mathrm{d} v
\end{gathered}
$$

In it, $\mathrm{h}_{\mathrm{a}_{m n}, \mathrm{~b}^{\prime}}\left(\mathrm{x}^{\prime}, \mathrm{y}^{\prime}\right)$ is the scale of the mother wavelet and translation function, as shown below:

$$
\mathrm{h}_{\mathrm{a}_{m n}, \mathrm{~b}^{\prime}}\left(\mathrm{x}^{\prime}, \mathrm{y}^{\prime}\right)=\left(\mathrm{a}_{m} \mathrm{a}_{n}\right)^{-0.5} h\left(\frac{\mathrm{x}^{\prime}-b_{\mathrm{x}^{\prime}}}{\mathrm{a}_{m}}, \frac{\mathrm{y}^{\prime}-\mathrm{b}_{\mathrm{y}^{\prime}}}{\mathrm{a}_{n}}\right)
$$

$\left(\mathrm{a}_{m} \mathrm{a}_{n}\right)=\left(\mathrm{a}_{m}, \mathrm{a}_{n}\right)$ is discrete scale, $\mathrm{b}^{\prime}=\left(b_{\mathrm{x}^{\prime}}, \mathrm{b}_{\mathrm{y}^{\prime}}\right)$ is displacement scale.

Secondary fractional order wavelet reconstruction formula is:

$$
\begin{gathered}
\mathrm{f}(\mathrm{x}, \mathrm{y})=\frac{1}{C} \iint F\left\{\sum \sum \iint \frac{1}{\mathrm{a}_{m} \mathrm{a}_{n}} W\left(\mathrm{a}_{m n}, \mathrm{~b}^{\prime}\right) \times H\left(\mathrm{a}_{m} \mu, \mathrm{a}_{n} \nu\right) \exp \left(-\mathrm{j} 2 \pi \mu b_{\mathrm{x}^{\prime}},-\mathrm{j} 2 \pi \nu \mathrm{b}_{\mathrm{y}^{\prime}}\right) \times d b_{\mathrm{x}^{\prime}} d \mathrm{~b}_{\mathrm{y}^{\prime}}\right\} \\
\left(\mathrm{x}^{\prime}, \mathrm{y}^{\prime}\right) B_{-\rho 1,-\rho 2}\left(\mathrm{x}, \mathrm{y}, \mathrm{x}^{\prime}, \mathrm{y}^{\prime}\right) \mathrm{dx}^{\prime} \mathrm{dy}^{\prime}
\end{gathered}
$$

In image denoising, the pros and cons of various algorithms are ultimately comes down to the evaluation on the quality of the images, so you need a reasonable image quality assessment standards. Reference literature both at home and abroad, the evaluation standard is relatively uniform. After a lot of research and verification, the current widely used in scientific research personnel peak signal-to-noise ratio as evaluation indexes. Defined as: including noise image peak signal-to-noise ratio $\mathrm{p} 0$ and denoising image peak signal-to-noise ratio $(\mathrm{p} 1$, as shown below:

$$
\begin{gathered}
p 0=-10 \lg \left(\frac{\sum_{i=1}^{m} \sum_{j=1}^{n} N^{2}(\mathrm{i}, \mathrm{j})}{m \times n \times 255^{2}}\right) \\
p 1=-10 \lg \left(\frac{\sum_{i=1}^{m} \sum_{j=1}^{n}\left(\mathrm{X}(\mathrm{i}, \mathrm{j})-\mathrm{X}^{\prime}(\mathrm{i}, \mathrm{j})\right)^{2}}{m \times n \times 255^{2}}\right)
\end{gathered}
$$

$\mathrm{X}(\mathrm{i}, \mathrm{j})$ is primitive noise image, $X^{\prime}(\mathrm{i}, \mathrm{j})$ is for image denoising is estimated, $N(\mathrm{i}, \mathrm{j})$ is the estimated noise. After denoising images of $p 1$ value, the greater the denoising processing after the image more close to the original image, the image denoising effect is better.

Objective image quality index used peak signal to noise ratio of $p$ value, the optimal $p$ values for images, need to accurately estimate the residual noise, here is mainly to have 
nothing to do with the image of the zero mean Gauss white noise estimation. The input image is often has been polluted by the noise of the image, contains xmn and noise of the image noise standard deviation $a_{m n}$ is unknown, estimate of the image $\mathrm{P}$ values equivalent to the estimate of the standard deviation of $a_{m n}$.

Image noise estimation methods for the standard deviation of $a_{m n}$ [9]: subtract with noise image denoising image difference to estimate the value of the noise signal, this method needs to use effectively filter out noise and well keep the edge details of both filter, will contain noise image into several parts, respectively estimates that each part $a_{m n}$ , choose the more appropriate as $a_{m n}$ actual estimated values; To estimate the noise of the image by image statistical features of the standard deviation $a_{m n}$.

Based on two-dimensional fractional wavelet transform in image denoising, the optimal $\mathrm{p}$ value selection is a very important link. This paper puts forward a optimal $\mathrm{p}$ value selection method based on noise estimation, now define the input noise unknown root mean square error of the $\mathrm{M}$ and unknown input noise peak signal to noise ratio of $\mathrm{Pu}$ objective evaluation criteria for the new image denoising, such as formula (12) and (13) [11.12]:

$$
\begin{gathered}
M=\frac{1}{\mathrm{~m} \times \mathrm{n}} \sum_{i=1}^{m} \sum_{j=1}^{n}\left(\mathrm{f}(\mathrm{i}, \mathrm{j})-N(\mathrm{i}, \mathrm{j})-X^{\prime}(\mathrm{i}, \mathrm{j})\right)^{2} \\
P u=-10 \lg \frac{M}{255^{2}}
\end{gathered}
$$

among them, $f(i, j)$ is the original noise image, $X^{\prime}(i, j)$ is estimated after denoising, image noise $N(\mathrm{i}, \mathrm{j})$ is estimated.

$N(\mathrm{i}, \mathrm{j})$ approximates to the real value of noise, $\mathrm{f}(\mathrm{i}, \mathrm{j})-\mathrm{N}(\mathrm{i}, \mathrm{j})$ more close to the original image without noise, can be thought of as $f(i, j)-N(i, j)$ on the estimate of $X^{\prime}(\mathrm{i}, \mathrm{j})$, and the fractional wavelet transform denoising after $X^{\prime}(\mathrm{i}, \mathrm{j})$ can also be considered for $\mathrm{X}(\mathrm{i}, \mathrm{j})$ kind of estimate, by comparing two kinds of estimates have been the $\mathrm{Pu}$ can be used as $X^{\prime}(\mathrm{i}, \mathrm{j})$ standard to evaluate the gap between two estimates, $\mathrm{Pu}$, the greater the degree of approximation, the higher of the two, this time can be thought of $X^{\prime}(\mathrm{i}, \mathrm{j})$, p value is the most optimal, when $X^{\prime}(\mathrm{i}, \mathrm{j})=X(\mathrm{i}, \mathrm{j})$, Pu is equal to $\mathrm{p}$. And the $P$ value by the same token, the $\mathrm{M}$ value is smaller, the $\mathrm{Pu}$ value, the greater the $X^{\prime}(\mathrm{i}, \mathrm{j})$ and $X(\mathrm{i}, \mathrm{j})$ higher degree of approximation, show the better denoising effect. The optimal corresponding fractional time calculated $\mathrm{Pu}$ is the optimal estimates of the $\mathrm{p}$ values [13.14].

\section{Mixed Image Denoising Model}

Image processing mainly is the advantage of the characteristics of the image data can be sparse representation, which most of the image information can be represented by some important image information. For example of image wavelet transform, image most of the information may be expressed by the part of the wavelet coefficients, and this part of the wavelet coefficients can be through out most of the original image information. In wavelet denoising, due to this part of the wavelet coefficient represents the image of the important information, so the important wavelet coefficients are generally not for processing, and only need to the rest of the wavelet coefficient of correlation processing. Wavelet transform of the sparse sex is wavelet transform can effectively one of the reasons for image denoising. 
Though wavelet transform is a kind of more effective image denoising method, but wavelet transform in denoising of a fault that we have to improve. The disadvantage is that the image to use when processing the two-dimensional wavelet is the tensor product of one-dimensional wavelets, that wavelet transform can effectively deal with the image of some strange and not work very well in the image line strange or bizarre. Some rich texture images after wavelet denoising, the texture will be "the scales" phenomenon, but most of the images of nature with a certain number of singular line edge (details), so it is necessary to improve the shortcomings. Finite element algorithm and winding phenomenon, so in the inverse finite element method, the denoised image is found after denoising the general also needs to do to reduce the winding phenomenon.

In order to improve the denoising method based on finite element of the denoising effect is not ideal, this paper presents a kind of based on image block wavelet denoising collaboration with finite element method. The thinking of the denoising method is the first block of image; Then select one of the non homogenous blocks, denoising by using finite element method in order to protect the image texture information, and the homogenous blocks with wavelet denoising method to remove noise. In the experimental part as you can see, this method can get rid of the noise in the edge of the well save image texture information, very suitable for processing some rich texture image.

Homogenous block comes mainly from the concept of image noise variance estimation, is refers to the standard variance smaller image block, so the image block contains less texture information. Image noise estimation in terms of the concept of a homogenous block is often used, designed to determine the condition of homogenous blocks, selection of image in the homogenous blocks, to deal with these homogenous blocks to estimate the noise of the image.

Assuming that the image is divided into a certain scale and the overlap of each image block size, the smaller the variance of image block, according to the literature $\{10\}$, then the image block contains the texture information is less, so you can through the variance of image block compared to a threshold to determine whether the image block for homogeneity. To adjacent tiles standard variance of value as a threshold, in the original image Lena and add noise of Lena image as an example, the homogenous blocks judgment conditions, the effect of it can be seen that most of the homogenous blocks can be selected out.

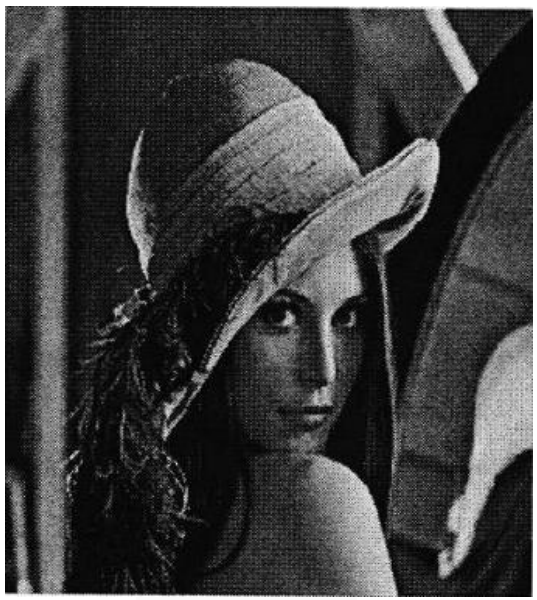

1. original image

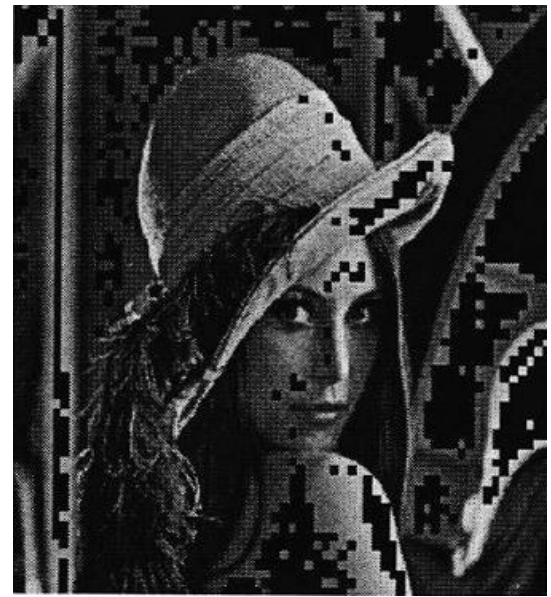

2. Image without homogenous block 


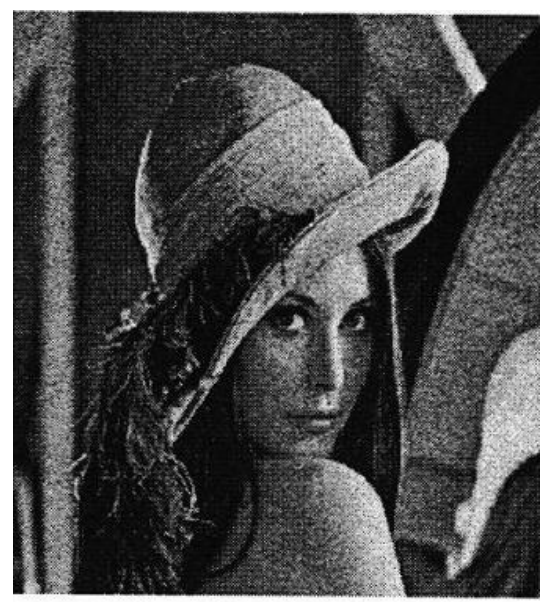

3. Image with noise

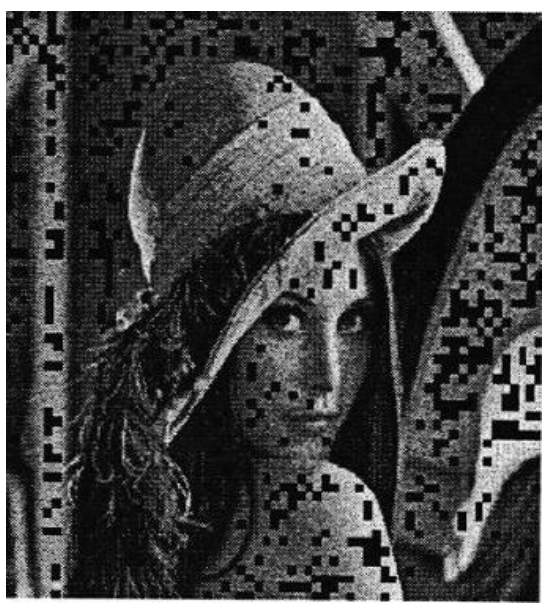

4. Noise image without homogenous block

Based on the selection of homogenous block condition, this section presents a wavelet joint denoising method and finite element method. Compared the denoising method, the basic idea is not homogeneous, smaller variance of homogenous blocks, homogenous blocks can be regarded as image smoothing area, the area of line singularity, which contain little images in a homogenous block contains the image edge details, such as line singularity using wavelet transform and the characteristics of the different finite element transformation denoising, can deal with homogenous blocks with wavelet transform, the finite element transformation process non homogenous block.

\section{Experiment and Analysis}

To illustrate the effectiveness of the proposed image denoising method, here to standard Lena image is commonly used in image processing, for example, choose several groups of different gaussian noise, with the finite element denoising, wavelet filtering denoising filtering method, to evaluate the practical value of denoising method.

Specific denoising process is: the impulse noise detection, according to the type (1) according to (2), (3) the impulse noise filtering, and then estimate the standard deviation of the residual noise. $a_{m n}$, then estimate the rest of the zero mean Gauss white noise, and then use the Pu optimal method to calculate the optimal order number $\mathrm{p}$, the optimal fractional wavelet domain filtered Gauss white noise. In this method, impulse noise detection adopts $7 \times 7$ window, according to the simulation experience value, took the four parameters $\mathrm{k}$, obtains the best filtering effect, take 5 parameter $\mathrm{t}$. The simulation experiment results of various methods for filtering are shown in table 1. Figure 2 is $a_{m n}$ variety of filter method for $a_{m n}=40$ pulse noise and $a_{m n}=40$ white noise filtering of the output image after mixed noise of Gauss.

Table 1. Lena Image Denoising $P$ Value Comparison (/db)

\begin{tabular}{|c|c|c|c|c|c|c|c|}
\hline Noise & $\begin{array}{c}\{20,20 \\
\}\end{array}$ & $\begin{array}{c}\{30,20 \\
\}\end{array}$ & $\begin{array}{c}\{40,30 \\
\}\end{array}$ & $\begin{array}{c}\{50,30 \\
\}\end{array}$ & $\begin{array}{c}\{40,30 \\
\}\end{array}$ & $\begin{array}{c}\{50,30 \\
\}\end{array}$ & $\begin{array}{c}\{60,30 \\
\}\end{array}$ \\
\hline $\begin{array}{c}\text { Noise of } \\
\text { image }\end{array}$ & 20.03 & 18.67 & 18.05 & 17.37 & 16.85 & 16.15 & 15.35 \\
\hline $\begin{array}{c}\text { Finite } \\
\text { element }\end{array}$ & 28.04 & 27.58 & 26.30 & 25.19 & 24.48 & 24.19 & 23.78 \\
\hline $\begin{array}{c}\text { Wavelet } \\
\text { transform }\end{array}$ & 27.63 & 27.19 & 26.39 & 26.03 & 25.40 & 25.01 & 24.29 \\
\hline $\begin{array}{c}\text { Method } \\
\text { in the } \\
\text { paper }\end{array}$ & 28.24 & 28.04 & 27.49 & 27.10 & 26.30 & 26.08 & 25.58 \\
\hline
\end{tabular}


The table 1 shows that different proportion and the size of the mixed noise background, the proposed hybrid filtering algorithm is used to remove the mixed noise in the image of the proceeds of the $\mathrm{P}$ value is the highest.

Mixed noise consists of impulse noise and Gauss white noise, as the change of noise ratio, the effect of various denoising algorithm strength is different. When impulse noise ratio is greater than the Gauss white noise, finite elementing alone effect is obviously enhanced, when the ratio is greater than the impulse noise and Gauss white noise, enhance the effect of wavelet transform, separate the effect of median filtering. Visible, finite element for noise suppression ability is used to filter out the narrow pulse signal is very effective, but for short tail distribution noise such as Gauss noise, finite element showed poor filtering performance.

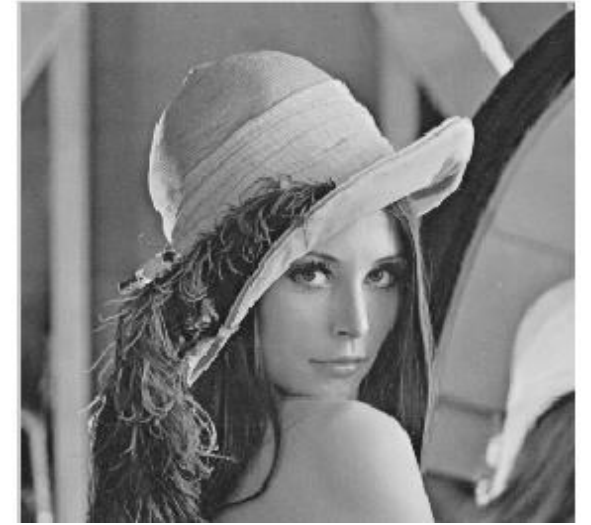

1. Noiseless image

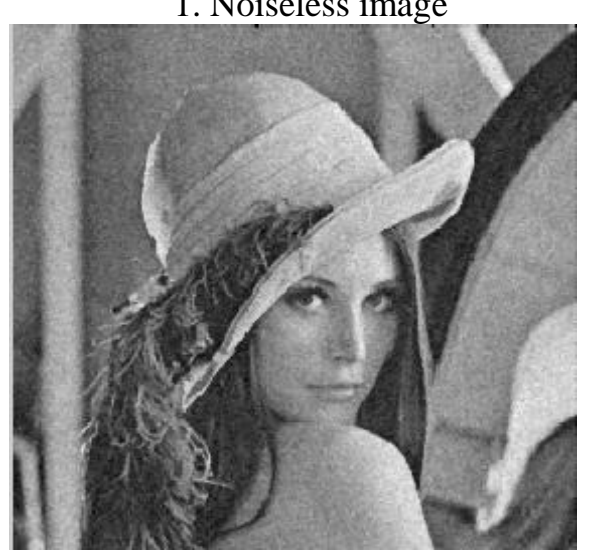

3. Image after a finite element denoising

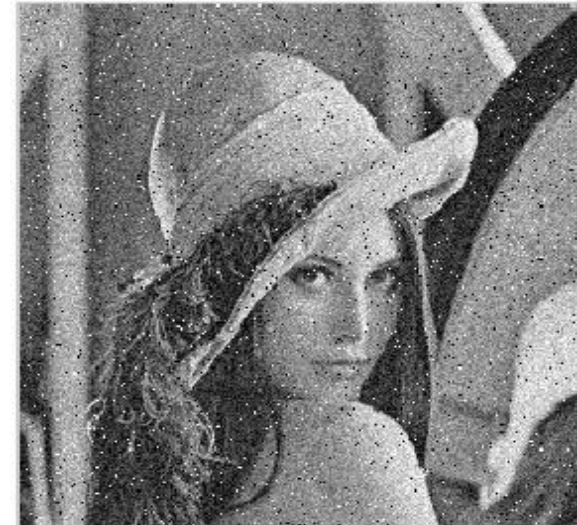

2. Containing mixed noise image

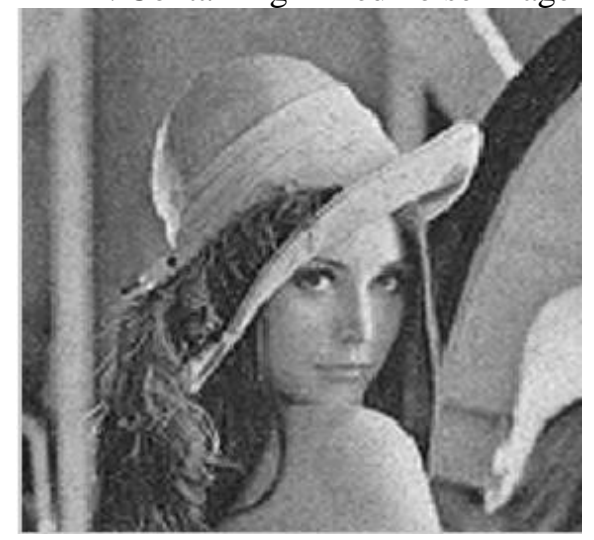

4. Image after the wavelet denoising

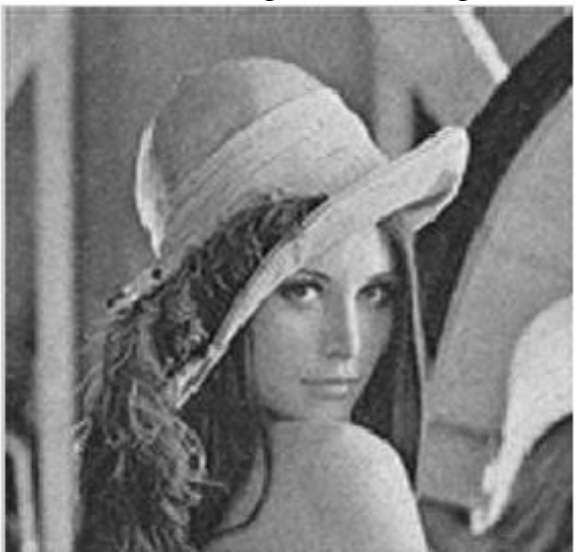

5. Image after the hybrid filter

Figure 2. Denoising of Lena Image 
By above knowable, this article uses multiple filter method is superior to single average filter or wavelet denoising method. Separate images become very smooth, after finite element and separate the wavelet denoising method is not able to completely filter to remove the impulse noise point, this paper adopts the hybrid algorithm of filtering effect is better than both.

To sum up, this paper is on the basis of the existing image denoising algorithms, performance improvement, and puts forward a new hybrid filtering algorithm, this algorithm can more reasonably selecting two-dimensional fractional wavelet transform, the optimal order estimates of the number of $p$, finally get good denoising effect, and it has certain actual application value.

\section{Conclusion}

In view of the actual application of multiple images of the unknown noise pollution, this paper puts forward the denoising algorithm is mainly using the wavelet transform is suitable for processing point strange and finite element transformation line is suitable for processing some strange characteristics, and adopt the method of image partition the image into homogeneous blocks and non homogenous blocks, use wavelet transform to process the homogenous blocks, rather than a homogenous block by finite element transformation processing, the method combining the finite element filter can effectively remove the impulse noise excellent properties and secondary fractional order threshold denoising method of wavelet filter in addition to good ability of gaussian noise, to a certain extent, to improve the image visual effect. The experimental results show that the method has a good denoising effect, should have good practical value.

\section{References}

[1] T. Huang, G. Yang and G. Tang, "A fast two-dimensional median filtering algorithm [J]", Acoustics, Speech and Signal Processing, IEEE Transactions on, vol. 27, no. 1, (2005), pp. 13-18.

[2] A C. Bovik, T S. Huang and D C. Munson Jr., "A generalization of median filtering using linear combinations of order statistics [J]", Acoustics, Speech and Signal Processing, IEEE Transactions on, vol. 31, no. 6, (2006), pp. 1342-1350.

[3] Y H. Lee and S A. Kassam, "Generalized median filtering and related nonlinear filtering techniques [J]", Acoustics, Speech and Signal Processing, IEEE Transactions on, vol. 33, no. 3, (2006), pp. 672-683.

[4] M. Antonini, M. Barlaud and P. Mathieu, "Image coding using wavelet transform [J]", Image Processing, IEEE Transactions on, vol. 1, no. 2, (2007), pp. 205-220.

[5] A. Grinsted, J C. Moore and S. Jevrejeva, "Application of the cross wavelet transform and wavelet coherence to geophysical time series [J]", Nonlinear processes in geophysics, vol. 11, nos. 5/6, (2004), pp. 561-566.

[6] H. Li, B S. Manjunath and S K. Mitra, "Multisensor image fusion using the wavelet transform [J]", Graphical models and image processing, vol. 57, no. 3, (2005), pp. 235-245.

[7] A S. Lewis and G. Knowles, "Image compression using the 2-D wavelet transform [J]", Image Processing, IEEE Transactions on, vol. 1, no. 2, (2006), pp. 244-250.

[8] O. Rockinger, "Image sequence fusion using a shift-invariant wavelet transform [C]", Image Processing, 2005, Proceedings, International Conference on. IEEE, vol. 3, (2005), pp. 288-291.

[9] S G. Chang, B. Yu and M. Vetterli, "Adaptive wavelet thresholding for image denoising and compression [J]”, Image Processing, IEEE Transactions on, vol. 9, no. 9, (2000), pp. 1532-1546.

[10] A. Buades, B. Coll and J M. Morel, "A review of image denoising algorithms, with a new one [J]", Multiscale Modeling \& Simulation, vol. 4, no. 2, (2005), pp. 490-530.

[11] J L. Starck, E J. Candès and D L. Donoho, "The curvelet transform for image denoising [J]", Image Processing, IEEE Transactions on, vol. 11, no. 6, (2002), pp. 670-684.

[12] J. Portilla, V. Strela and M J. Wainwright, "Image denoising using scale mixtures of Gausss in the wavelet domain [J]”, Image Processing, IEEE Transactions on, vol. 12, no. 11, (2003), pp. 1338-1351.

[13] M. Elad and M. Aharon, "Image denoising via sparse and redundant representations over learned dictionaries [J]", Image Processing, IEEE Transactions on, vol. 15, no. 12, (2006), pp. 3736-3745.

[14] K. Dabov, A. Foi and V. Katkovnik, "Image denoising by sparse 3-D transform-domain collaborative filtering [J]”, Image Processing, IEEE Transactions on, vol. 16, no. 8, (2007), pp. 2080-2095. 


\section{Author}

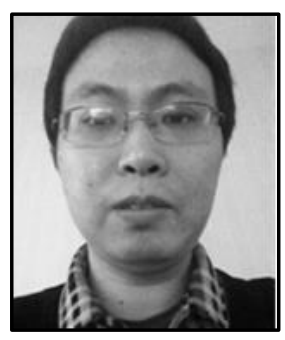

Zeng Tai-sheng he received the BS and MS degrees from the HuaQiao University, China, in 1998 and 2005. His main research interests include image engineering (image processing, image analysis, image understanding and technique application) 
International Journal of Signal Processing, Image Processing and Pattern Recognition Vol.8, No.10 (2015) 